\title{
Efficient and Large-Scale Synthesis of Few-Layered Graphene Using an Arc-Discharge Method and Conductivity Studies of the Resulting Films
}

\author{
Yingpeng Wu, Bin Wang, Yanfeng Ma, Yi Huang, Na Li, Fan Zhang, and Yongsheng Chen ( $\varangle)$ \\ Key Laboratory of Functional Polymer Materials and Center for Nanoscale Science \& Technology, Institute of Polymer Chemistry, College \\ of Chemistry, Nankai University, Tianjin 300071, China \\ Received: 10 May 2010 / Revised: 29 June 2010 / Accepted: 3 August 2010 \\ C The Author(s) 2010. This article is published with open access at Springerlink.com
}

\begin{abstract}
An arc-discharge method using a buffer gas containing carbon dioxide has been developed for the efficient and large-scale synthesis of few-layered graphene. The resulting samples of few-layered graphene, well-dispersed in organic solvents such as $\mathrm{N}, \mathrm{N}$-dimethylformamide (DMF) and 1,2-dichlorobenzene (o-DCB), were examined by transmission electron microscopy (TEM), X-ray diffraction (XRD), Raman spectroscopy, atomic force microscopy (AFM), and thermal gravimetric analysis (TGA). The electrical conductivity and transparency of flexible films prepared using a direct solution process have also been studied.
\end{abstract}

\section{KEYWORDS}

Graphene, arc-discharge, few-layered, film, conductivity

\section{Introduction}

While graphene has been studied experimentally for over 40 years [1,2], worldwide interest has been generated only recently as its unique structure and excellent chemical, physical and mechanical properties have become apparent [3-6]. This has been summarized in a very recent comprehensive review of various graphene materials [1]. Three main methods are available for producing this material. Of these, micromechanical cleavage of graphite [3,4]-although producing graphene with excellent quality-is unsuitable for large-scale preparation. Growth on $\mathrm{SiC}$ [7] or other substrates [8,9] suffers from the same problem of low output. Chemical exfoliation is an effective way to produce graphene in large quantities [10-16]. However, chemical exfoliation involves complex chemical processes, and it generally affords graphene sheets with heavily functionalized organic groups (defects) and, thus, low conductivity. Furthermore, it has been proposed-and subsequently experimentally demonstrated-that the properties of graphene materials depend on the number of graphene layers present [17]. Thus, efforts have been made to synthesize few- or multi-layered graphene materials with a predetermined number of layers and fewer defects. Recently, new methods have been employed to prepare graphene sheets with one, two or more layers, such as arc-discharge techniques [18-20], chemical vapor deposition [21-23], microwave irradiation [24], solvothermal-assisted exfoliation [25] and bottom-up methods [26]. Similar to the synthesis

Address correspondence to yschen99@nankai.edu.cn 
of carbon nanotubes, the arc-discharge method is used for producing large-scale graphene sheets with fewer defects (functional groups). Subrahmanyan et al. obtained graphene sheets with two to four layers by arc-discharge in a mixture of hydrogen and helium [18]. In addition, Wu et al. prepared graphene sheets by helium arc discharge [20], and Li et al. prepared $\mathrm{N}$-doped multi-layered graphene sheets by arc discharge with a mixture of $\mathrm{NH}_{3}$ and $\mathrm{He}$ as the buffer gas [19]. In this work, we describe a highly efficient and large-scale arc-discharge process in a buffer of carbon dioxide and helium for the synthesis of few-layered graphene sheets with high quality. The method is relatively safe and inexpensive compared with techniques already reported in the literature, and more importantly, the graphene sheets have much better electrical conductivity and can be well dispersed in common organic solvents such as $\mathrm{N}, \mathrm{N}$ dimethylformamide (DMF) and 1,2-dichlorobenzene (o-DCB); this makes the material processable via solution methods for film and device fabrication.

\section{Experimental}

\subsection{Synthesis of few-layered graphene sheets}

Briefly, a direct current (DC) arc-discharge was carried out in a home-made water-cooled stainless steel chamber filled with a mixture of carbon dioxide and helium. Different buffer gas compositions, ranging from $5 \mathrm{vol} \%$ to $40 \mathrm{vol} \% \mathrm{CO}_{2}$, and direct currents of 100 to $200 \mathrm{~A}$ were employed for the purposes of comparison. The discharge voltage was kept around $30 \mathrm{~V}$ by controlling the distance between the two electrodes. Both electrodes were normal graphite rods obtained commercially, with an anode diameter of $13 \mathrm{~mm}$ and a cathode diameter of $40 \mathrm{~mm}$. After discharge, the cotton-like deposition product that formed on the inner wall of the chamber was collected and examined. The optimized conditions for the production of fewlayered graphene were found to be as follows: a low voltage $(<35 \mathrm{~V})$, high buffer gas pressure $(>1270$ Torr), high current (about $150 \mathrm{~A}$ ) and $25 \%-40 \%(v / v) \mathrm{CO}_{2}$ in the total buffer gas. In a typical run, tens of grams of high-quality graphene sheets with four to five layers can be generated in minutes.

\subsection{Instruments and methods}

Transmission electron microscopy (TEM) images were obtained using a Tecnai 20 microscope with an accelerating voltage of $200 \mathrm{kV}$. X-ray diffraction (XRD) measurements were carried out on a Rigaku D/Max2500 diffractometer with $\mathrm{Cu} \mathrm{K \alpha}$ radiation. Raman spectra were recorded with a Renishaw in Via Raman spectrometer using laser excitation at $514.5 \mathrm{~nm}$. Tapping-mode atomic force microscopy (AFM) measurements were performed using a Multimode SPM from Digital Instruments with a Nanoscope IIIa Controller. Samples for AFM images were prepared by spin-coating a dispersion of graphene in DMF $(0.35 \mathrm{mg} / \mathrm{mL})$ onto a freshly cleaved mica surface (500 rpm for $18 \mathrm{~s}$ followed by $1500 \mathrm{rpm}$ for $60 \mathrm{~s}$ ) and allowing it to dry in air. Thermal gravimetric analysis (TGA) was carried out using a NETZSCH STA 409PC analyzer. The heating rate was $5{ }^{\circ} \mathrm{C} / \mathrm{min}$ from room temperature to $1000{ }^{\circ} \mathrm{C}$ in an $\mathrm{N}_{2}$ atmosphere. X-ray photoelectron spectroscopy (XPS) was used to investigate defects in the few-layered graphene. XPS spectra were recorded using a Kratos Axis Ultra DLD spectrometer employing a monochromatic $\mathrm{Al} \mathrm{K \alpha} \mathrm{X}$-ray source $(h v=1486.6 \mathrm{eV})$, hybrid (magnetic/electrostatic) optics and a multi-channel plate and delay line detector. Electrical measurements were recorded using a Keithley SCS 4200 semiconductor characterization section. UV-vis spectra were recorded using a Jasco V-570 spectrometer. The samples for UV-vis spectroscopy were prepared by dispersing the desired amount of graphene in DMF with about 5 min of mild sonication.

\section{Results and discussion}

In the TEM image shown in Fig. 1(a), one can see that transparent graphene sheets are present over a large area with high purity. The size of the sheets was measured using about 50 TEM images. The geometric means of the length and width of each sheet were used to define their size, which was in the range 100-300 nm (Fig. 1(c)), and aspect ratio, which was generally over 100, consistent with the literature [18-20]. We attempted to obtain an electron diffraction image for a bulk sample using TEM, but no clear diffraction pattern 

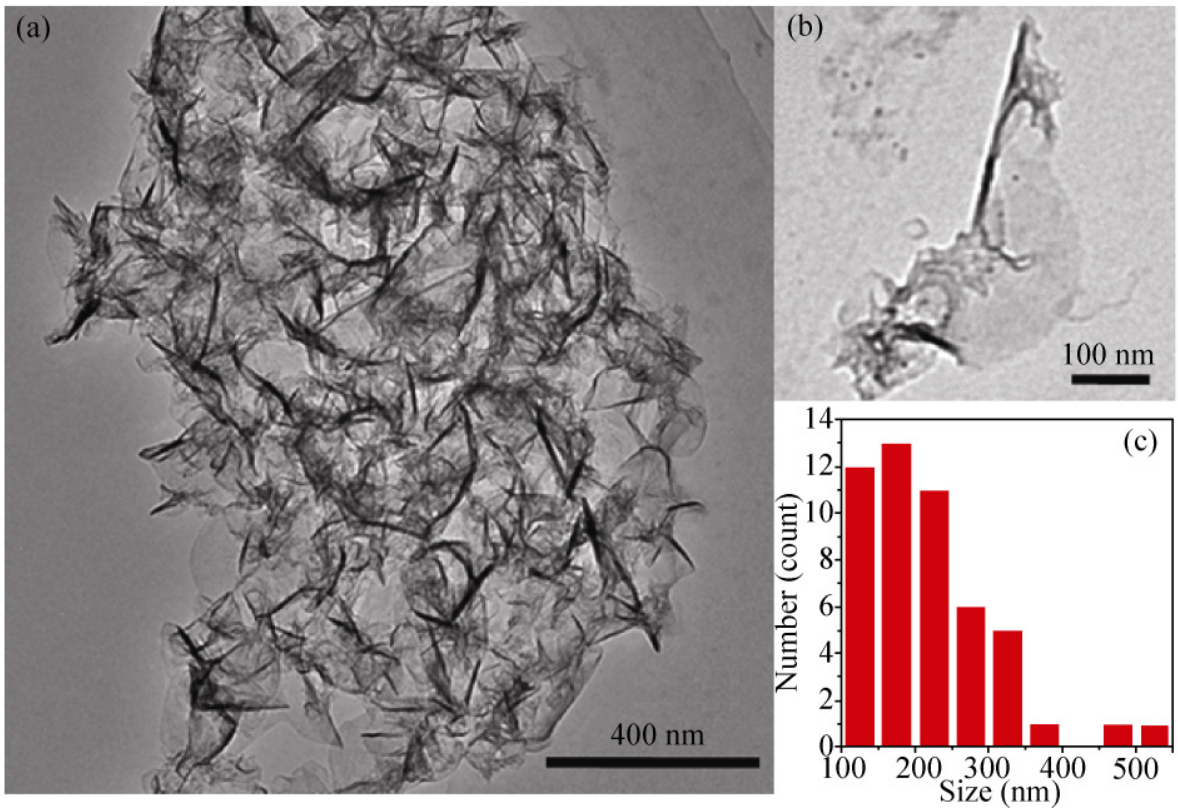

Figure 1 (a) Low- and (b) high-magnification TEM images of graphene sheets produced using the optimized conditions. (c) Histograms of the size distribution of 50 images obtained by TEM

was observed due to the very limited long-range order in the bulk sample. It is important to note that the surfaces of the graphene sheets are not perfectly flat, with wrinkles over the surface giving different levels of transparency (Fig. 1(b)). The high-resolution TEM (HRTEM) image (Fig. 2) shows folded regions (Fig. 2(a)) and a scrolled edge (Fig. 2(b)), and the number of graphene layers can be clearly distinguished [26]. This particular TEM image shows that the graphene sheets consist of four (arrow L), five (M), three $(\mathrm{N})$ and three $(\mathrm{O})$ layers. More TEM images are shown in Fig. S-1 in the Electronic Supplementary Material (ESM).

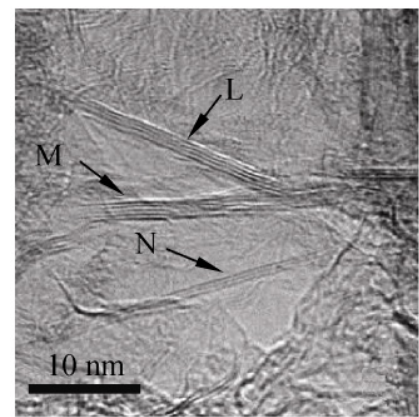

(a)

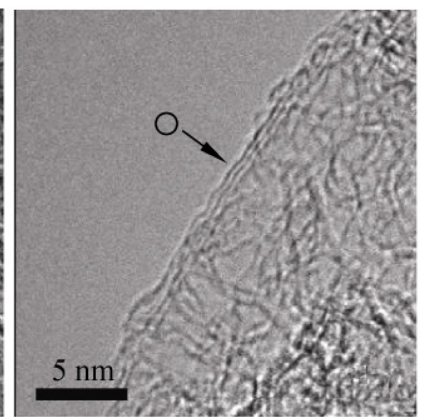

(b)
Figure 2 HRTEM images of (a) folded regions and (b) a scrolled edge. The graphene sheets consist of four (arrow L), five (M), three $(\mathrm{N})$ and three $(\mathrm{O})$ layers
XRD was used to characterize the structure of the few-layered graphene sheets, as shown in Fig. 3. There are two main peaks in the XRD pattern. The sharp peak at $2 \theta=26.3^{\circ}$ indicates a highly organized crystal structure with an interlayer spacing of $0.339 \mathrm{~nm}$, which is consistent with the layer spacing of normal graphite. This occurs because the interlayer spacing inside the few-layered graphene sheet has a structure similar to that of normal graphite. The broad peak at $2 \theta=21.1^{\circ}$, corresponding to a $d$-spacing of $0.417 \mathrm{~nm}$, is indicative of a lower degree of crystallization and the presence of some defects that were possibly caused by the presence of $\mathrm{CO}_{2}$. This suggests the existence of regions of expanded stacking of more corrugated or disordered graphene sheets, probably existing at the edge areas [27, 28]. This is consistent with the TEM results given above. In addition to the main peaks, there is a rather weak and broad peak at $2 \theta=11.3^{\circ}$, corresponding to a $d$-spacing of $0.777 \mathrm{~nm}$. This peak is similar to the typical diffraction peak of graphene oxide (GO) [13] and is a possible indication of the presence of inter-few-layered graphene (containing defects). Another very weak shoulder at $2 \theta=25.7^{\circ}$ on the main peak at $2 \theta=26.3^{\circ}$, corresponding to a $d$-spacing of $0.344 \mathrm{~nm}$, may arise from another species. However, based on experimental and simulated results [27], it 


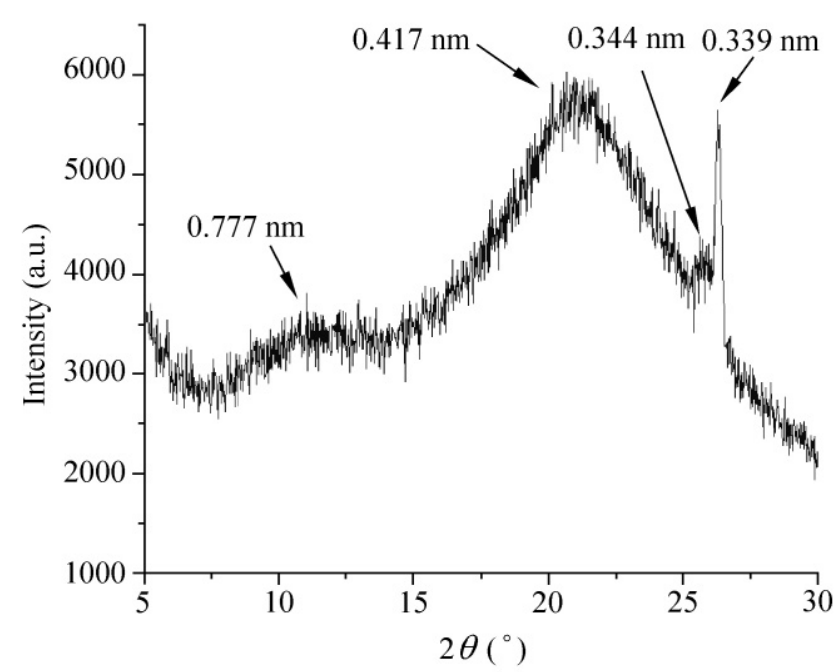

Figure 3 XRD pattern of the few-layered graphene sheets. The sharp peak at $2 \theta=26.3^{\circ}$ indicates a highly organized crystal structure with an interlayer spacing of $0.339 \mathrm{~nm}$. The broad peak at $2 \theta=21.1^{\circ}$ is indicative of a material with lower crystallinity and some defects. The peak at $2 \theta=11.3^{\circ}$ may be due to diffraction by the inter-few-layered graphene, since it is similar to the typical diffraction peak of GO

could also be due to the complicated nature of the size, curvature, sheet rotation, and atom positioning in the graphene sheets.

Raman spectroscopy is a rapid and easy way to obtain an indication of the structure and quality of carbon materials. Figure 4 shows a typical Raman spectrum of our product. The three intense features are the $\mathrm{D}$ band at $1334 \mathrm{~cm}^{-1}$, the $\mathrm{G}$ band at $1577 \mathrm{~cm}^{-1}$ and the $2 \mathrm{D}$ band at $2662 \mathrm{~cm}^{-1}$. The $\mathrm{D}$ band is associated with disorder arising from structural defects and is very weak in a single graphene sheet but its intensity increases with the number of layers present [29]. The $G$ band corresponds to the zone-center $E_{2 g}$ mode related to phonon vibrations in $\mathrm{sp}^{2}$ carbon domains [30]. The ratio of the intensity of the $G$ band to that of the $D$ band $\left(I_{\mathrm{G}} / I_{\mathrm{D}}\right)$ is related to the in-plane crystallite size $L_{a}$ and a value of $L_{a}=5.9 \mathrm{~nm}$ was obtained using the equation $L_{\mathrm{a}}(\mathrm{nm})=4.4\left(I_{\mathrm{G}} / I_{\mathrm{D}}\right)$ [29]. The $2 \mathrm{D}$ band is very sensitive to the number of layers in the sample. As shown in Fig. 4, our few-layered graphene sheets show a single Lorentzian profile, as in the case of few-layered graphene sheets obtained using the CVD method [21], but different from the case of few-layered graphene sheets generated by microcleaving highly ordered pyrolytic graphite (HOPG)- [21]. The reason behind this is probably the same as that in the case of graphene sheets generated using the CVD method, where the same levels of ordered stacking and/or electronic coupling between graphene layers do not occur in all regions of the graphene sheets, in contrast to the graphene sheets generated using the HOPG method [21]. The intensity ratio $I_{\mathrm{G}} / I_{2 \mathrm{D}}$ also has a good correlation with the number of graphene layers present. The $I_{\mathrm{G}} / I_{2 \mathrm{D}}$ ratio of our product is $\sim 1.03$, which is comparable to the value for three layers of CVD-grown graphene $\left(I_{\mathrm{G}} / I_{2 \mathrm{D}} \sim 1.3\right)$ [21]. However, we found that our few-layered graphene sheets show an average line width of $\sim 85 \mathrm{~cm}^{-1}$, larger than that obtained by other methods [21,31]. We believe that there are at least two reasons for this. Firstly, there are probably more defects in our few-layered graphene sheets due to edge oxidation by $\mathrm{CO}_{2}$. Secondly, it may be that a mixture of graphene sheets with different numbers of layers were used in our measurement, whereas the earlier measurements were carried out with individual graphene sheets [21, 31].

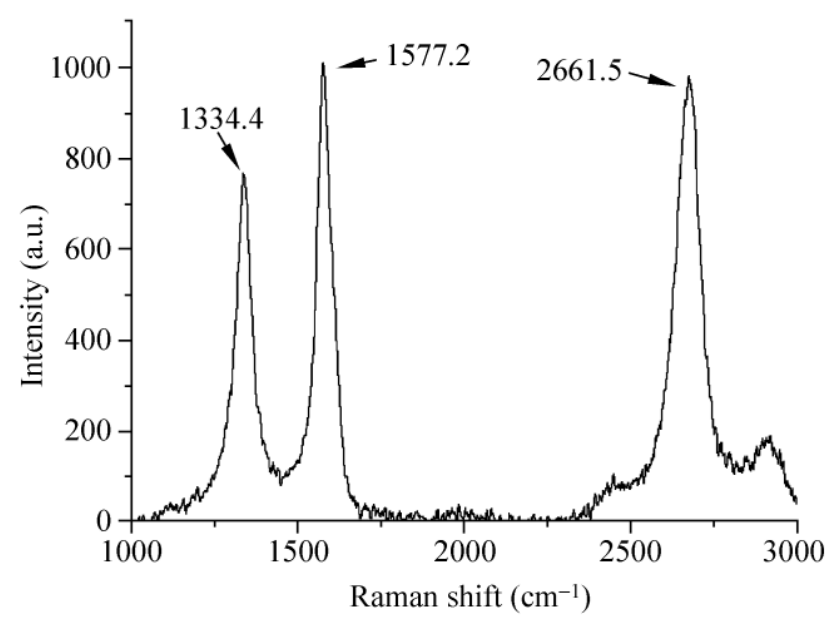

Figure 4 A typical Raman spectrum of few-layered graphene obtained by the arc-discharge process. The three intense features are the $\mathrm{D}$ band at $1334 \mathrm{~cm}^{-1}$, the $\mathrm{G}$ band at $1577 \mathrm{~cm}^{-1}$ and the $2 \mathrm{D}$ band at $2662 \mathrm{~cm}^{-1}$. An in-plane crystallite size $L_{\mathrm{a}}=5.9 \mathrm{~nm}$ was calculated using the equation $L_{\mathrm{a}}(\mathrm{nm})=4.4\left(I_{\mathrm{G}} / I_{\mathrm{D}}\right)$. The intensity ratio $I_{\mathrm{G}} / I_{2 \mathrm{D}}$ of the product is $\sim 1.03$, which is comparable to the value for three layers of CVD-grown graphene $\left(I_{\mathrm{G}} / I_{2 \mathrm{D}} \sim 1.3\right)$

We also used a large number of AFM images to study the thickness distribution of the graphene sheets generated using our arc-discharge method (Fig. 5). 


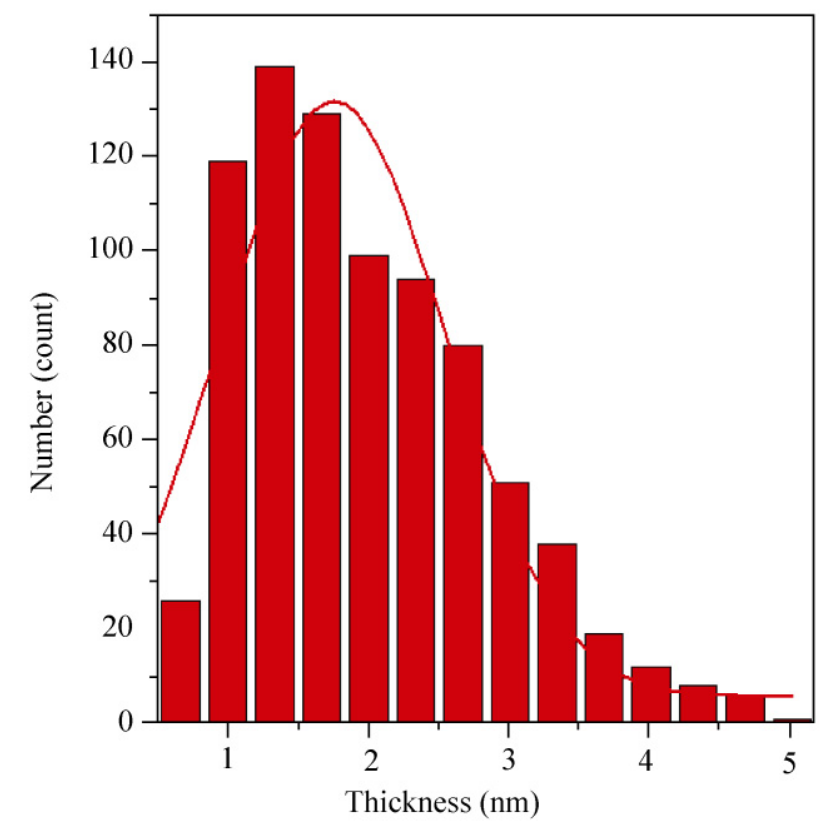

Figure 5 The height of the graphene sheets exhibits a Gaussian distribution, and the maximum in the height distribution is $\sim 1.76 \mathrm{~nm}$, based on an analysis of more than 800 graphene sheets using AFM images

The height of the graphene films exhibited a Gaussian distribution, and the maximum in the height distribution was $1.76 \mathrm{~nm}$ based on an AFM statistical height analysis for more than 800 sheets. This indicates that the maximum in the distribution of the graphene layers is four to five. Some typical AFM images used in this statistical analysis are shown in Fig. S-2 in the ESM.

Figure 6 shows the TGA curve of the few-layered graphene sheets. To calculate the weight loss of our graphene product in TGA and compare it with that of GO produced by chemical methods, we selected two temperature regions: (1) under $100{ }^{\circ} \mathrm{C}$ (normally the region of solvent weight loss in the case of GO) and (2) $100-300{ }^{\circ} \mathrm{C}$ (mainly the region of weight loss associated with functional groups in the case of GO). There is hardly any weight loss under $100{ }^{\circ} \mathrm{C}$ and a small weight loss of $\sim 1.2 \%$ in the range $100-300{ }^{\circ} \mathrm{C}$, where GO experiences a major loss in mass [32]. These results indicate that, although there may be some functional groups present due to the $\mathrm{CO}_{2}$ buffer gas, the number of functional groups on these few-layered graphene sheets is much less than that in samples prepared using chemical methods [32].

X-ray photoelectron spectroscopy (XPS) was used to estimate the number of defects in the few-layered graphene. High-resolution C 1s XPS spectra are shown in Fig. 7. Deconvolution of the spectra indicated that the relative content of the non-oxygenated ring $\mathrm{C}$ atoms $(284.8 \mathrm{eV}$ ) was $84 \%$, that of $\mathrm{C}$ atoms in $\mathrm{C}-\mathrm{O}$ bonds $(286.3 \mathrm{eV})$ was $11 \%$, and that of carboxylate $\mathrm{C}$ atoms $(\mathrm{O}-\mathrm{C}=\mathrm{O}, 288.9 \mathrm{eV})$ was $5 \%$. The defects in the sample arise from oxidization by $\mathrm{CO}_{2}$ in the buffer gas, and the XPS data indicate that the relative content of the non-oxygenated ring $\mathrm{C}$ atoms is much higher than that in conventional GO obtained using chemical methods [33]. We also ran an elemental analysis for

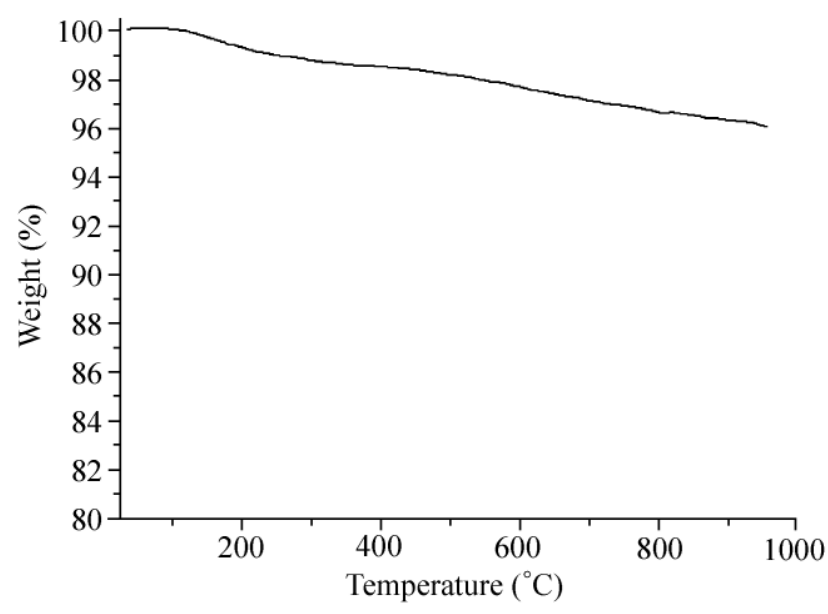

Figure 6 TGA curve of the few-layered graphene sheets. The heating rate was $5{ }^{\circ} \mathrm{C} / \mathrm{min}$ from room temperature to $1000{ }^{\circ} \mathrm{C}$ in an $\mathrm{N}_{2}$ atmosphere

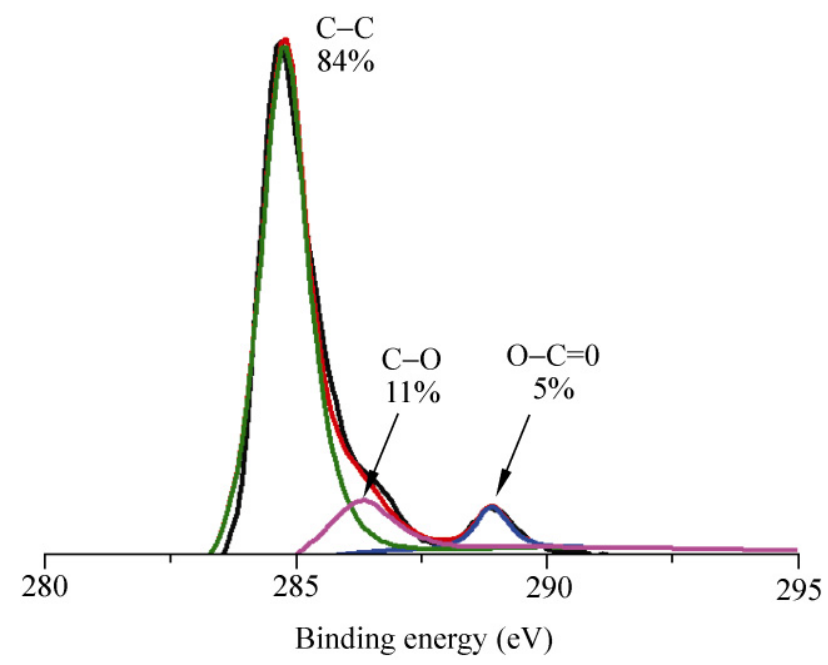

Figure 7 High-resolution C 1s XPS spectra of the few-layered graphene; deconvolution indicated that the relative content of the non-oxygenated ring $\mathrm{C}$ atoms $(284.8 \mathrm{eV})$ was $84 \%, \mathrm{C}$ atoms in $\mathrm{C}-\mathrm{O}$ bonds $(286.3 \mathrm{eV})$ was $11 \%$ and carboxylate $\mathrm{C}$ atoms $(\mathrm{O}-\mathrm{C}=\mathrm{O}$, $288.9 \mathrm{eV}$ ) was $5 \%$ 
our few-layered graphene products which indicated a composition $97.02 \% \mathrm{C}, 0.40 \% \mathrm{H}$, and $2.58 \% \mathrm{O}$. This indicates, overall, a much lower percentage of $\mathrm{sp}^{3}$ carbon atoms and fewer defects than that indicated by XPS, which is consistent with the TGA results discussed above. We suspect that the larger defect or oxygen content indicated by XPS may have the following three causes: (1) large inherent errors are present in quantitative analysis by XPS; (2) the effective sampling depth of XPS is only $2-5 \mathrm{~nm}$, and since the functional groups are presumably mainly on the surface of our product, the XPS surface analysis could be very different from that of the elemental (bulk) analysis; (3) some solvent or water adsorption may occur on graphene due to its large surface area and surface energy.

Solution processing capability is a key for low-cost processes in device and composite applications [34, 35].
Figure 8(a) shows the absorption spectra of solutions of the few-layered graphene with different concentrations in DMF. The straight line shown in Fig. 8(b) is a linear least-squares fit to the data with an $R$ value of 0.992 , which indicates that the few-layered graphene is homogeneously dissolved in the solvent. The absorption peak of the few-layered graphene is at $270 \mathrm{~nm}$, considerably red-shifted as compared to the absorption peak of GO at $226 \mathrm{~nm}$ [32]. This is as expected, given the smaller number of functional groups and defects present and the lower extent of disruption of the $\pi$-conjugation in the few-layered graphene in comparison to that in GO [32]. Moreover, as a result of their excellent solution processability, we were able to directly fabricate a variety of films using the organic solutions as precursors. The films, which were prepared by spin-coating a substrate with dispersions of fewlayered graphene in DMF $(0.7 \mathrm{mg} / \mathrm{mL})$, were then dried

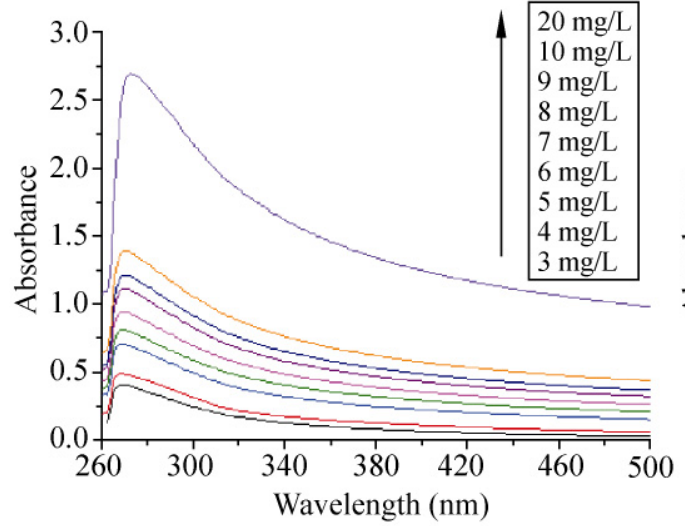

(a)

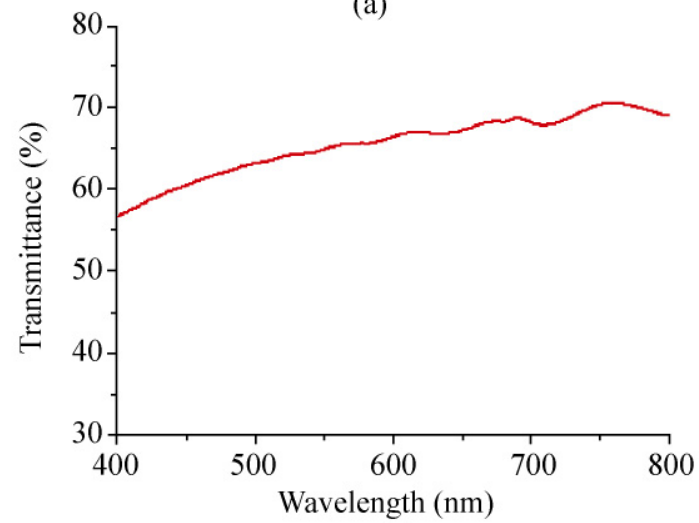

(c)

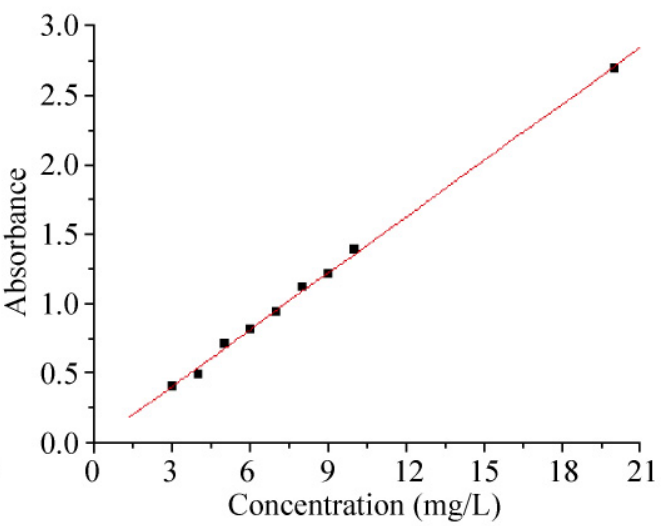

(b)

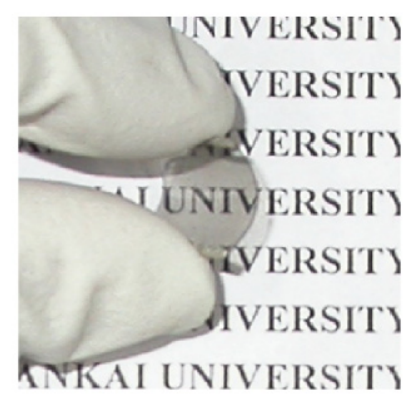

(d)

Figure 8 (a) Concentration dependence of the UV absorption of few-layered graphene in DMF (with concentrations of 3-20 mg/L). (b) The straight line is a linear least-squares fit to the data, which indicates that the few-layered graphene was homogeneously dissolved in the solvent. (c) The optical transmittance spectrum of the film on a PET substrate shows a transmittance of $\sim 65 \%$ at $550 \mathrm{~nm}$ with a square film resistance of $\sim 670 \mathrm{k} \Omega / \mathrm{sq}$. (d) An image of a few-layered graphene film on a flexible PET substrate 
for electrical conductivity measurements. The currentvoltage $(I-V)$ characteristics of the films are shown in Fig. 9. The classical linear $I-V$ relationship is indicative of semiconducting electrical conductivity with an electrical conductivity of $\sim 11 \mathrm{~S} / \mathrm{cm}$ (thickness $\sim 25 \mathrm{~nm}$ ). Furthermore, a flexible conductive film was also made by drop-casting a solution of graphene in $o$-DCB $(4 \mathrm{mg} / \mathrm{mL})$ on a polyethylene terephthalate (PET) film followed by drying in vacuum at room temperature. The square resistance of the film was $\sim 670 \mathrm{k} \Omega / \mathrm{sq}$ with a transmittance of $\sim 65 \%$ at $550 \mathrm{~nm}$ (Fig. 8(c)). More importantly, the conductivity was retained quite well after the film was bent at an angle of $\sim 45^{\circ}$ more than 1500 times (Fig. 8(d)). Further studies examining the suitability of these films as electrodes in different devices are currently underway in our laboratory.

\section{Conclusions}

We have developed a method for synthesizing fewlayered graphene by arc discharge in a mixture of carbon dioxide and helium. The resulting few-layered graphene sheets have fewer defects than those generated using chemical methods, and more importantly, they can be easily dispersed in organic solvents for subsequent solution processing. Flexible and highly conductive films can be directly fabricated by spincoating or drop-casting of their solutions. This com-

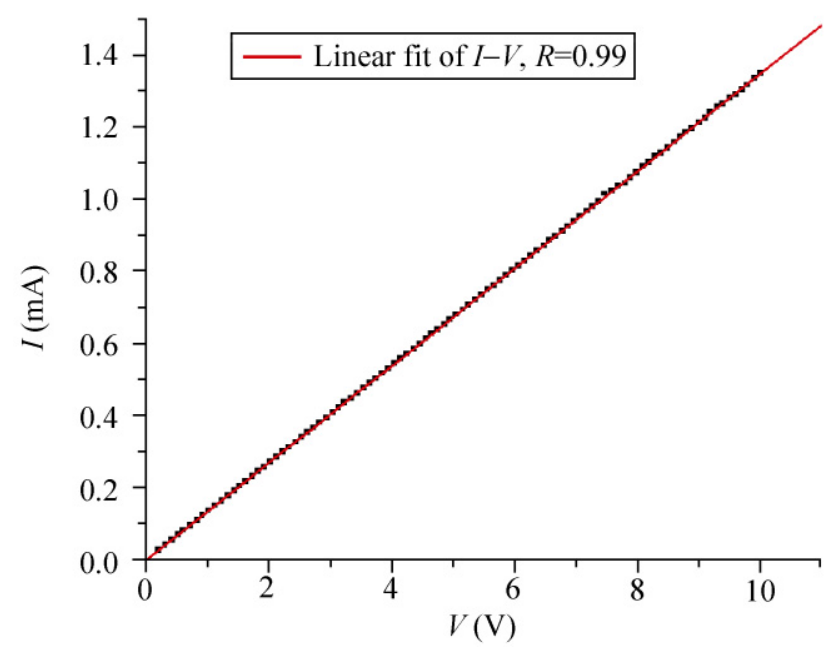

Figure 9 Current-voltage $(I-V)$ curve of a graphene film that was prepared by the dispersion of few-layered graphene in DMF $(0.7 \mathrm{mg} / \mathrm{mL})$ using spin-coating. The thickness was $\sim 25 \mathrm{~nm}$, and the electrical conductivity was $\sim 11 \mathrm{~S} / \mathrm{cm}$ bination of properties should make this material a good candidate for use in the fabrication of electrodes in various devices and as electrical fillers for conducting composite materials.

\section{Acknowledgements}

The authors gratefully acknowledge financial support from the National Natural Science Foundation of China (NSFC) (Nos. 50933003, 20774047, 50903044, 50902073), the Chinese Ministry of Science and Technology (MOST) (No. 2009AA032304), and the Natural Science Foundation (NSF) of Tianjin Municipality (No. 08JCZDJC25300).

Electronic Supplementary Material: TEM and AFM images are available in the online version of this article at http://dx.doi.org/10.1007/s12274-010-0027-3 and are accessible free of charge.

Open Access: This article is distributed under the terms of the Creative Commons Attribution Noncommercial License which permits any noncommercial use, distribution, and reproduction in any medium, provided the original author(s) and source are credited.

\section{References}

[1] Zhu, Y. W.; Murali, S.; Cai, W. W.; Li, X. S.; Suk, J. W.; Potts, J. R.; Ruoff, R. S. Graphene and graphene oxide: Synthesis, properties, and applications. Adv. Mater., in press, DOI: 10.1002/adma.201001068.

[2] May, J. W. Platinum surface LEED rings. Surf. Sci. 1969, 17, 267-270.

[3] Lu, X. K.; Yu, M. F.; Huang, H.; Ruoff, R. S. Tailoring graphite with the goal of achieving single sheets. Nanotechnology 1999, 10, 269-272.

[4] Novoselov, K. S.; Geim, A. K.; Morozov, S. V.; Jiang, D.; Zhang, Y.; Dubonos, S. V.; Grigorieva, I. V.; Firsov, A. A. Electric field effect in atomically thin carbon films. Science 2004, 306, 666-669.

[5] Novoselov, K. S.; Geim, A. K.; Morozov, S. V.; Jiang, D.; Katsnelson, M. I.; Grigorieva, I. V.; Dubonos, S. V.; Firsov, A. A. Two-dimensional gas of massless Dirac fermions in graphene. Nature 2005, 438, 197-200. 
[6] Stankovich, S.; Dikin, D. A.; Dommett, G. H. B.; Kohlhaas, K. M.; Zimney, E. J.; Stach, E. A.; Piner, R. D.; Nguyen, S. T.; Ruoff, R. S. Graphene-based composite materials. Nature 2006, 442, 282-286.

[7] Berger, C.; Song, Z. M.; Li, X. B.; Wu, X. S.; Brown, N.; Naud, C.; Mayou, D.; Li, T. B.; Hass, J.; Marchenkov, A. N.; Conrad, E. H.; First, P. N.; de Heer, W. A. Electronic confinement and coherence in patterned epitaxial graphene. Science 2006, 312, 1191-1196.

[8] Chae, S. J.; Gunes, F.; Kim, K. K.; Kim, E. S.; Han, G. H.; Kim, S. M.; Shin, H. J.; Yoon, S. M.; Choi, J. Y.; Park, M. H.; Yang, C. W.; Pribat, D.; Lee, Y. H. Synthesis of large-area graphene layers on poly-nickel substrate by chemical vapor deposition: Wrinkle formation. Adv. Mater. 2009, 21, 2328-2333.

[9] Li, X. S.; Cai, W. W.; An, J. H.; Kim, S.; Nah, J.; Yang, D. X.; Piner, R.; Velamakanni, A.; Jung, I.; Tutuc, E.; Banerjee, S. K.; Colombo, L. Large-area synthesis of high-quality and uniform graphene films on copper foils. Science 2009, 324, 1312-1314.

[10] Niyogi, S.; Bekyarova, E.; Itkis, M. E.; McWilliams, J. L.; Hamon, M. A.; Haddon, R. C. Solution properties of graphite and graphene. J. Am. Chem. Soc. 2006, 128, 7720-7721.

[11] Stankovich, S.; Dikin, D. A.; Piner, R. D.; Kohlhaas, K. A.; Kleinhammes, A.; Jia, Y.; Wu, Y.; Nguyen, S. T.; Ruoff, R. S. Synthesis of graphene-based nanosheets via chemical reduction of exfoliated graphite oxide. Carbon 2007, 45, 1558-1565.

[12] Schniepp, H. C.; Li, J. L.; McAllister, M. J.; Sai, H.; Herrera-Alonso, M.; Adamson, D. H.; Prud'homme, R. K.; Car, R.; Saville, D. A.; Aksay, I. A. Functionalized single graphene sheets derived from splitting graphite oxide. $J$. Phys. Chem. B 2006, 110, 8535-8539.

[13] McAllister, M. J.; Li, J. L.; Adamson, D. H.; Schniepp, H. C.; Abdala, A. A.; Liu, J.; Herrera-Alonso, M.; Milius, D. L.; Car, R.; Prud'homme, R. K.; Aksay, I. A. Single sheet functionalized graphene by oxidation and thermal expansion of graphite. Chem. Mater. 2007, 19, 4396-4404.

[14] Yu, A. P.; Ramesh, P.; Itkis, M. E.; Bekyarova, E.; Haddon, R. C. Graphite nanoplatelet-epoxy composite thermal interface materials. J. Phys. Chem. C 2007, 111, 7565-7569.

[15] Li, X. L.; Wang, X. R.; Zhang, L.; Lee, S. W.; Dai, H. J. Chemically derived, ultrasmooth graphene nanoribbon semiconductors. Science 2008, 319, 1229-1232.

[16] Li, D.; Muller, M. B.; Gilje, S.; Kaner, R. B.; Wallace, G. G. Processable aqueous dispersions of graphene nanosheets.
Nat. Nanotechnol. 2008, 3, 101-105.

[17] Geim, A. K.; Novoselov, K. S. The rise of graphene. Nat. Mater. 2007, 6, 183-191.

[18] Subrahmanyam, K. S.; Panchakarla, L. S.; Govindaraj, A.; Rao, C. N. R. Simple method of preparing graphene flakes by an arc-discharge method. J. Phys. Chem. C 2009, 113, 4257-4259.

[19] Li, N.; Wang, Z.; Zhao, K.; Shi, Z.; Gu, Z.; Xu, S. Large scale synthesis of $\mathrm{N}$-doped multi-layered graphene sheets by simple arc-discharge method. Carbon 2010, 48, 255-259.

[20] Wu, Z. S.; Ren, W. C.; Gao, L. B.; Zhao, J. P.; Chen, Z. P.; Liu, B. L.; Tang, D. M.; Yu, B.; Jiang, C. B.; Cheng, H. M. Synthesis of graphene sheets with high electrical conductivity and good thermal stability by hydrogen arc discharge exfoliation. ACS Nano 2009, 3, 411-417.

[21] Reina, A.; Jia, X. T.; Ho, J.; Nezich, D.; Son, H. B.; Bulovic, V.; Dresselhaus, M. S.; Kong, J. Large area, few-layer graphene films on arbitrary substrates by chemical vapor deposition. Nano Lett. 2009, 9, 30-35.

[22] Kim, K. S.; Zhao, Y.; Jang, H.; Lee, S. Y.; Kim, J. M.; Kim, K. S.; Ahn, J. H.; Kim, P.; Choi, J. Y.; Hong, B. H. Large-scale pattern growth of graphene films for stretchable transparent electrodes. Nature 2009, 457, 706-710.

[23] Reina, A.; Thiele, S.; Jia, X.; Bhaviripudi, S.; Dresselhaus, M. S.; Schaefer, J. A.; Kong, J. Growth of large-area singleand bi-layer graphene by controlled carbon precipitation on polycrystalline Ni surfaces. Nano Res. 2009, 2, 509-516.

[24] Janowska, I.; Chizari, K.; Ersen, O.; Zafeiratos, S.; Soubane, D.; Da Costa, V.; Speisser, V.; Boeglin, C.; Houllé, M.; Bégin, D.; Plee, D.; Ledoux, M. -J.; Pham-Huu, C. Microwave synthesis of large few-layer graphene sheets in aqueous solution of ammonia. Nano Res. 2010, 3, 126-137.

[25] Qian, W.; Hao, R.; Hou, Y.; Tian, Y.; Shen, C.; Gao, H.; Liang, X. Solvothermal-assisted exfoliation process to produce graphene with high yield and high quality. Nano Res. 2009, 2, 706-712.

[26] Meyer, J. C.; Geim, A. K.; Katsnelson, M. I.; Novoselov, K. S.; Booth, T. J.; Roth, S. The structure of suspended graphene sheets. Nature 2007, 446, 60-63.

[27] Li, Z. Q.; Lu, C. J.; Xia, Z. P.; Zhou, Y.; Luo, Z. X-ray diffraction patterns of graphite and turbostratic carbon. Carbon 2007, 45, 1686-1695.

[28] Srinivas, G.; Zhu, Y.; Piner, R.; Skipper, N.; Ellerby, M.; Ruoff, R. Synthesis of graphene-like nanosheets and their hydrogen adsorption capacity. Carbon 2010, 48, 630-635. 
[29] Subrahmanyam, K. S.; Vivekchand, S. R. C.; Govindaraj, A.; Rao, C. N. R. A study of graphenes prepared by different methods: Characterization, properties and solubilization. $J$. Mater. Chem. 2008, 18, 1517-1523.

[30] Ferrari, A. C.; Meyer, J. C.; Scardaci, V.; Casiraghi, C.; Lazzeri, M.; Mauri, F.; Piscanec, S.; Jiang, D.; Novoselov, K. S.; Roth, S.; Geim, A. K. Raman spectrum of graphene and graphene layers. Phys. Rev. Lett. 2006, 97, 187401.

[31] Graf, D.; Molitor, F.; Ensslin, K.; Stampfer, C.; Jungen, A.; Hierold, C.; Wirtz, L. Raman imaging of graphene. Solid State Commun. 2007, 143, 44-46.

[32] Zhang, L.; Liang, J.; Huang, Y.; Ma, Y.; Wang, Y.; Chen, Y. Size-controlled synthesis of graphene oxide sheets on a large scale using chemical exfoliation. Carbon 2009, 47, 3365-3368.

[33] Becerril, H. A.; Mao, J.; Liu, Z.; Stoltenberg, R. M.; Bao, Z.; Chen, Y. Evaluation of solution-processed reduced graphene oxide films as transparent conductors. ACS Nano 2008, 2, 463-470.

[34] Xu, Y.; Wang, Y.; Liang, J.; Huang, Y.; Ma, Y.; Wan, X.; Chen, Y. A hybrid material from graphene and PEDOT with high conductivity, flexibility and transparency. Nano Res. 2009, 2, 343-348.

[35] Xu, Y.; Long, G.; Huang, L.; Huang, Y.; Wan, X.; Ma, Y.; Chen, Y. Polymer photovoltaic devices with transparent graphene electrodes produced by spin-casting. Carbon 2010, 48, 3308-3311. 